\title{
Fundamental media considerations for the propagation of phase-conjugate waves
}

\author{
Amnon Yariv \\ California Institute of Technology, Pasadena, California 91125
}

Received April 17, 1991

\begin{abstract}
Rigorous and approximate conditions that need to be satisfied by a propagation medium to enable phase conjugation to occur are derived. It is shown that, in spite of the fact that in general, losses spoil phase conjugation, in the important case of paraxial beam propagation (along $z$ ), a $z$-dependent loss can be tolerated. In addition, nonlinear losses (gain) and nonlinear dielectrics are also permitted under some fairly general circumstances.
\end{abstract}

Phase-conjugate optics deals with the generation, by four-wave mixing, of monochromatic time-reversed replicas of input waves. These waves propagate in a direction opposite that of the input and experience, in reverse, the same history. ${ }^{1,2}$ If the input wave is expressed as

$$
\mathbf{E}_{1}(\mathbf{r}, t)=\operatorname{Re}\left[\mathbf{E}_{1}(\mathbf{r}) e^{i \omega t}\right]
$$

then the output wave must be of the form of

$$
\begin{aligned}
\mathbf{E}_{2}(\mathbf{r}, t)=\operatorname{Re}\left[\mathbf{E}_{2}(\mathbf{r}) e^{i \omega t}\right] & =\operatorname{Re}\left[C \mathbf{E}_{1}{ }^{*}(\mathbf{r}) e^{i \omega t}\right] \\
& =\operatorname{Re}\left[C^{*} \mathbf{E}_{1}(\mathbf{r}) e^{i \omega(-t)}\right],
\end{aligned}
$$

where $C$ is any (complex) constant.

In practice we know how to generate a phaseconjugate replica $E_{2}$ of $E_{1}$, so that $\mathbf{E}_{2}(\mathbf{r})=C \mathbf{E}_{1}{ }^{*}(\mathbf{r})$ over only some limited region in space, say, in the vicinity of $z=0$, where $z$ is the nominal axis of propagation. The question then arises whether $E_{2}$ is still the phase conjugate of $E_{1}$ throughout the halfspace $z<0$. In this case the two, counterpropagating, waves would possess everywhere identical wave fronts, and any aberrations or distortions suffered by $E_{1}$ are thus healed in the reverse propagation of $E_{2}$.

In what the follows we inquire about the set of conditions that the propagation medium needs to satisfy to ensure perfect or approximate phase conjugation.

Consider an input wave with electric and magnetic fields $\mathbf{E}_{1}$ and $\mathbf{H}_{1}$. [In what follows, all field quantities will refer to spatially dependent complex amplitudes unless specified otherwise, i.e., $\mathbf{E}_{1}=$ $\mathbf{E}_{1}(\mathbf{r})$, etc.] $\mathbf{E}_{1}$ and $\mathbf{H}_{1}$ obey Maxwell's equations

$$
\begin{aligned}
& \nabla \times \mathbf{E}_{1}=-i \omega \mu \mathbf{H}_{1}, \\
& \nabla \times \mathrm{H}_{1}=\sigma \mathbf{E}_{1}+i \omega \epsilon \mathbf{E}_{1}
\end{aligned}
$$

or, equivalently, their complex conjugates

$$
\begin{aligned}
& \nabla \times \mathbf{E}_{1}{ }^{*}=i \omega \mu^{*} \mathbf{H}_{1}{ }^{*}, \\
& \nabla \times \mathbf{H}_{1}{ }^{*}=\sigma^{*} \mathbf{E}_{1}{ }^{*}-i \omega \epsilon^{*} \mathbf{E}_{1}{ }^{*} .
\end{aligned}
$$

If the field $\mathbf{E}_{2}, \mathbf{H}_{2}$ is a time-reversed version of $\mathbf{E}_{1}$, $\mathbf{H}_{1}$, it must satisfy

$$
\mathbf{E}_{2}=C \mathbf{E}_{1}^{*}, \quad \mathbf{H}_{2}=-C \mathbf{H}_{1}^{*},
$$

where $C$ is any complex constant. Use of Eqs. (5) in Eqs. (4) leads to

$$
\begin{aligned}
& \nabla \times \mathbf{E}_{2}=-i \omega \mu^{*} \mathbf{H}_{2}, \\
& \nabla \times \mathbf{H}_{2}=-\sigma^{*} \mathbf{E}_{2}+i \omega \epsilon^{*} \mathbf{E}_{2} .
\end{aligned}
$$

But since, in addition to being a time-reversed replica of $\mathbf{E}_{1}, \mathbf{H}_{1}$, the field $\mathbf{E}_{2}, \mathbf{H}_{2}$ is also a freely propagating wave, it must satisfy Maxwell's equations on its own; that is,

$$
\begin{aligned}
& \nabla \times \mathbf{E}_{2}=-i \omega \mu \mathbf{H}_{2}, \\
& \nabla \times \mathbf{H}_{2}=\sigma \mathbf{E}_{2}+i \omega \epsilon \mathbf{E}_{2} .
\end{aligned}
$$

For Eqs. (5) and (6) to be satisfied simultaneously the following conditions must hold:

$$
\begin{aligned}
\mu & =\mu^{*}, \\
\sigma & =-\sigma^{*}, \\
\epsilon & =\epsilon^{*},
\end{aligned}
$$

i.e., for true phase-conjugate propagation $\mu$ and $\epsilon$ need to be real, while $\sigma=0$ : no gain or loss in the medium.

It is important to see whether we can relax conditions (8)-(10) when the transmission and the distorting medium are lossy, i.e., when $\sigma \neq 0$. We consider the case of near-planar (paraxial) propagation along some (say, $z$ ) axis:

$$
E_{1}(\mathbf{r})=A_{1}(\mathbf{r}) e^{-i k z},
$$

where

$$
k \equiv \omega \sqrt{\mu \epsilon}, \quad \epsilon(\mathbf{r})=\epsilon+\delta(\mathbf{r}) .
$$

The quantity $\delta(\mathbf{r})$ accounts for index perturbations such as may be caused by material inhomogeneities in the optical path. $A_{1}(\mathbf{r})$ satisfies the paraxial Helmholtz equation [derivable from Eqs. (3)]

$$
\begin{array}{r}
\nabla_{t}^{2} A_{1}-2 i k \frac{\partial A_{1}}{\partial z}-k^{2} A_{1}+\omega^{2} \mu \epsilon(\mathbf{r}) A_{1}-i \omega \mu \sigma A_{1} \\
=0,
\end{array}
$$

where, self-consistently with the paraxial approximation, we left out terms involving $\nabla(\nabla \cdot \mathbf{E})$ and 
$\partial^{2} A_{1} / \partial z^{2}$. When Eq. (12) is used in Eq. (13), the latter simplifies to

$$
\nabla_{t}^{2} A_{1}-2 i k \frac{\partial A_{1}}{\partial z}+\omega^{2} \mu \delta(\mathbf{r}) A_{1}-i \omega \mu \sigma A_{1}=0 .
$$

The complex conjugate of Eq. (14) is

$$
\nabla_{t}^{2} A_{1}^{*}+2 i k \frac{\partial A_{1}^{*}}{\partial z}+\omega^{2} \mu^{*} \delta^{*}(\mathbf{r}) A_{1}^{*}+i \omega \mu^{*} \sigma^{*} A_{1}^{*}=0
$$

A wave

$$
E_{2}(\mathbf{r})=A_{2}(\mathbf{r}) e^{i k z},
$$

which is the phase conjugate of $\mathbf{E}_{1}$, satisfies, by definition,

$$
A_{2}(\mathbf{r})=C A_{1}^{*}(\mathbf{r}),
$$

which, when substituted into Eq. (15), gives

$$
\nabla_{t}^{2} A_{2}+2 i k \frac{\partial A_{2}}{\partial z}+\omega^{2} \mu^{*} \delta^{*}(r) A_{2}+i \omega \mu^{*} \sigma^{*} A_{2}=0 .
$$

The wave $A_{2}$, being a freely propagating wave, must, in addition, satisfy the paraxial wave equation. The equation in this case is identical to Eq. (14) except that, in view of Eq. (16), we replace $k$ by $-k$ :

$$
\nabla_{t}^{2} A_{2}+2 i k \frac{\partial A_{2}}{\partial z}+\omega^{2} \mu \delta(\mathbf{r}) A_{2}-i \omega \mu \sigma A_{2}=0 .
$$

We find, not surprisingly, that for $A_{2}$ to satisfy Eqs. (17) and (18) simultaneously the same set of conditions [Eqs. (8)-(10)] must be satisfied. Taking $\epsilon$ (and therefore $\delta$ ), $\sigma$, and $\mu$ as real quantities, we find that true phase conjugation requires that $\sigma=0$, i.e., no losses or gain. Intuitive reasoning suggests that the condition $\sigma=0$ might be too strict and that propagation through a medium with a loss (or gain) that depends only on $z$, i.e., $\sigma=\sigma(z)$, might still permit phase conjugation. To check this possibility we substitute into Eq. (14)

$$
A_{1}(\mathbf{r})=a_{1}(\mathbf{r}) \exp \left[-\int_{0}^{z} \alpha\left(z^{\prime}\right) \mathrm{d} z^{\prime}\right]
$$

and into Eq. (18)

$$
A_{2}(\mathbf{r})=a_{2}(\mathbf{r}) \exp \left[-\int_{z}^{L} \alpha\left(z^{\prime}\right) \mathrm{d} z^{\prime}\right] .
$$

The substitution of Eqs. (19) and (20) into (14) and (18) (in the case of real $\alpha, \sigma, \mu$, and $\delta$ ) leads to

$$
\begin{aligned}
\nabla_{t}^{2} a_{1}-2 i k \frac{\partial a_{1}}{\partial z}+ & \omega^{2} \mu \delta(\mathbf{r}) a_{1} \\
& +i[2 k \alpha(z)-\omega \mu \dot{\sigma}(z)] a_{1}=0 \\
\nabla_{t}^{2} a_{1}^{*}+2 i k \frac{\partial a_{1}^{*}}{\partial z} & +\omega^{2} \mu \delta(\mathbf{r}) a_{1}^{*} \\
& -i[2 k \alpha(z)-\omega \mu \sigma(z)] a_{1}^{*}=0 \\
\nabla_{t}^{2} a_{2}+2 i k \frac{\partial a_{2}}{\partial z}+ & \omega^{2} \mu \delta(\mathbf{r}) a_{2} \\
& +i[2 k \alpha(z)-\omega \mu \sigma(z)] a_{2}=0 .
\end{aligned}
$$

We find that if we choose $\alpha(z)=\omega \mu \sigma(z) / 2 k$, our wave equation will contain no $\sigma$, the last terms in Eqs. (21) drop out, and $a_{2} \propto a_{1}{ }^{*}$ is a valid solution. Since it was the last term involving $\sigma$ in Eqs. (17) and (18) that spoiled the phase conjugation, we have thus shown that, in the case of losses that depend only on $z$, phase conjugation still obtains since the actual fields $A_{1}$ and $A_{2}$ differ from $a_{1}$ and $a_{2}$, respectively, by $z$-dependent scale factors only.

Next we explore the possibility of phase conjugation in media with $\epsilon$ and $\sigma$ that are nonlinear, i.e., depend on the field intensity.

We consider the paraxial wave equation

$$
\nabla^{2} \mathbf{E}-i \omega \mu \sigma \mathbf{E}+\omega^{2} \mu \epsilon(\mathbf{r}) \mathbf{E}=0,
$$

where

$$
\begin{aligned}
\epsilon(\mathbf{r}) & =\epsilon+\delta \epsilon(\mathbf{r})+a(z)|E|^{2}, \\
\sigma(\mathbf{r}) & =\sigma_{0}(\mathbf{z})-b(z)|E|^{2} .
\end{aligned}
$$

We take the electric field as the sum of two oppositely propagating (scalar) waves:

$$
\begin{aligned}
E & =A_{1}(\mathbf{r}) e^{-i k z}+A_{2}(\mathbf{r}) e^{i k z}, \\
k^{2} & \equiv \omega^{2} \mu \epsilon .
\end{aligned}
$$

Our task will be to find out the conditions under which

$$
A_{2}(\mathbf{r}) \propto C(z) A_{1}(\mathbf{r})^{*},
$$

which signifies phase conjugation. The term $|E|^{2}$, which will figure prominently through Eqs. (24), takes the form

$$
|E|^{2}=\left|A_{1}\right|^{2}+\left|A_{2}\right|^{2}+A_{1} A_{2}{ }^{*} e^{-i 2 k z}+A_{1}{ }^{*} A_{2} e^{i 2 k z} .
$$

We distinguish between two main categories of media: The first is diffusive media, such as liquids and gases, in which strong diffusion smooths out the fine grating (whose period is $\lambda / 2$ ), represented by the last two terms in Eq. (28). The second category includes materials that can support the fine-grained grating (an example is saturated absorption in a crystal). We refer to this case as nondiffusive. To treat the effect of losses we put, as in Eqs. (19) and (20),

$$
\begin{aligned}
& A_{1}(\mathbf{r})=a_{1}(\mathbf{r}) \exp \left[-\int_{0}^{z} \alpha_{1}\left(z^{\prime}\right) \mathrm{d} z^{\prime}\right], \\
& A_{2}(\mathbf{r})=a_{2}(\mathbf{r}) \exp \left[-\int_{z}^{L} \alpha_{2}\left(z^{\prime}\right) \mathrm{d} z^{\prime}\right] .
\end{aligned}
$$

$\alpha_{1}(z)$ and $\alpha_{2}(z)$ are to be determined by substituting Eqs. (24) into Eq. (23), using Eqs. (25), (26), (29), and (30) and separating the resulting equation into a term involving exp $(-i k z)$ and another involving $\exp (i k z)$ :

$$
\begin{array}{r}
\left(\nabla_{t}^{2}+2 i k \frac{\partial}{\partial z}+\omega^{2} \mu \delta \epsilon+a \omega^{2} \mu\left[\left(\begin{array}{l}
1 \\
2
\end{array}\right)\left|A_{1}\right|^{2}+\left|A_{2}\right|^{2}\right]\right. \\
\left.+i\left\{2 k \alpha_{2}-\omega \mu \sigma_{0}+\omega \mu \mathrm{b}\left[\left(\begin{array}{l}
1 \\
2
\end{array}\right)\left|A_{1}\right|^{2}+\left|A_{2}\right|^{2}\right]\right\}\right) a_{2} \\
=0, \quad(31)
\end{array}
$$




$$
\begin{array}{r}
\left(\nabla_{t}^{2}+2 i k \frac{\partial}{\partial z}+\omega^{2} \mu \delta \epsilon+a \omega^{2} \mu\left[\left|A_{1}\right|^{2}+\left(\begin{array}{l}
1 \\
2
\end{array}\right)\left|A_{2}\right|^{2}\right]\right. \\
\left.-i\left\{2 k \alpha_{1}-\omega \mu \sigma_{0}+\omega \mu b\left[\left|A_{1}\right|^{2}+\left(\begin{array}{l}
1 \\
2
\end{array}\right)\left|A_{2}\right|^{2}\right]\right\}\right) a_{1}{ }^{*} \\
=0,
\end{array}
$$

where

$$
\left(\begin{array}{l}
1 \\
2
\end{array}\right)=\left\{\begin{array}{ll}
1 & \text { in diffusive media } \\
2 & \text { in nondiffusive media }
\end{array}\right. \text {. }
$$

We are now in a position to consider some special cases.

Case 1: No loss, nonlinear $\epsilon$. We put $\sigma_{0}=b=$ 0; Eqs. (31) and (32) become

$$
\begin{aligned}
\left\{\nabla_{t}^{2}+2 i k \frac{\partial}{\partial z}\right. & +\omega^{2} \mu \delta \epsilon(\mathbf{r}) \\
& \left.+a(z) \omega^{2} \mu\left[\left(\begin{array}{l}
1 \\
2
\end{array}\right)\left|A_{1}\right|^{2}+\left|A_{2}\right|^{2}\right]\right\} a_{2}=0 \\
\left\{\nabla_{t}^{2}+2 i k \frac{\partial}{\partial z}\right. & +\omega^{2} \mu \delta \epsilon(\mathbf{r}) \\
& \left.+a(z) \omega^{2} \mu\left[\left|A_{1}\right|^{2}+\left(\begin{array}{l}
1 \\
2
\end{array}\right)\left|A_{2}\right|^{2}\right]\right\} a_{1}^{*}=0 .
\end{aligned}
$$

In the diffusive case, $\left(\frac{1}{2}\right)=1$, the two equations above are identical, and phase conjugation obtains. In the nondiffusive case, $\left(\frac{1}{2}\right)=2$, the last two terms, which are first multiplied by $a(z)$, are dissimilar, and phase conjugation does not obtain. The exception is the case $\left|A_{1}(\mathbf{r})\right|^{2}=\left|A_{2}(\mathbf{r})\right|^{2}$, which is satisfied when the phase-conjugate mirror used to launch the phase-conjugate wave back into the medium under consideration has a reflectivity whose magnitude $|R|=1$. The above results were stated earlier. ${ }^{3}$

Case 2: Nonlinear $\epsilon$, linear loss $(b=0)$. Equations (31) and (32) become

$$
\begin{aligned}
\left\{\nabla_{t}^{2}+2 i k \frac{\partial}{\partial z}+\right. & \omega^{2} \mu \delta \epsilon(\mathbf{r})+a \omega^{2} \mu\left[\left(\begin{array}{l}
1 \\
2
\end{array}\right)\left|A_{1}\right|^{2}+\left|A_{2}\right|^{2}\right] \\
& \left.+i\left[2 k \alpha_{2}-\omega \mu \sigma_{0}(z)\right]\right\} a_{2}=0, \quad(34) \\
\left\{\nabla_{t}^{2}+2 i k \frac{\partial}{\partial z}+\right. & \omega^{2} \mu \delta \epsilon(\mathbf{r})+a \omega^{2} \mu\left[\left|A_{1}\right|^{2}+\left(\begin{array}{l}
1 \\
2
\end{array}\right)\left|A_{2}\right|^{2}\right] \\
- & \left.i\left[2 k \alpha_{1}-\omega \mu \sigma_{0}(z)\right]\right\} a_{1}^{*}=0 .
\end{aligned}
$$

In this case phase conjugation is possible only in the strong-diffusion case, $\left(\frac{1}{2}\right)=1$, since, when $\alpha_{1}=$ $\alpha_{2}=\omega \mu \sigma_{0}(z) / 2 k$ is chosen, Eqs. (34) and (35) become identical.

Case 3: Nonlinear $\epsilon$, nonlinear loss. This is the most general case and is described by Eqs. (31) and (32). In the case of no diffusion, no phase conjugation is possible, as the nonlinear dielectric constants, the terms involving $a$, seen by each wave are different. In the diffusive case, however, $\left(\frac{1}{2}\right)=1$, the two equations for $a_{2}$ and $a_{1}^{*}$ become identical provided that

$$
\begin{array}{r}
\alpha_{1}(z)=\alpha_{2}(z)=\frac{\omega \mu}{2 k}\left[\sigma_{0}(z)-b(z)\left(\left|A_{1}(x, y, z)\right|^{2}\right.\right. \\
\left.\left.+\left|A_{2}(x, y, z)\right|^{2}\right)\right] .
\end{array}
$$

Strictly speaking, Eq. (36) cannot be satisfied, since the left-hand side is a function of $z$ alone whereas the right-hand side depends on $x, y$, and $z$. In practice, however, if the nonlinear medium is in the far field, $\left.\left|A_{1}\right|(x, y, z)\right|^{2}$, for example, corresponds to the (spatial) spectral density of $A_{1}(x, y, z)$ and can in practice be a weak function of $x$ and $y$. To the extent that this is true, Eq. (33) can be satisfied, and phase conjugation obtains.

Case 4: Linear $\epsilon$, nonlinear loss. In this last case of interest, which applies to propagation through an amplifying or an absorbing medium at the resonant frequency so that the saturablemedium's contribution to $\epsilon$ is zero, we have

$$
\begin{aligned}
\left\{\nabla_{t}^{2}+2 i k \frac{\partial}{\partial z}+\right. & \omega^{2} \mu \delta \epsilon+i\left[2 k \alpha_{2}-\omega \mu \sigma_{0}+\omega \mu b\right] \\
& \left.\times\left[\left(\begin{array}{l}
1 \\
2
\end{array}\right)\left|A_{1}\right|^{2}+\left|A_{2}\right|^{2}\right]\right\} a_{2}=0, \\
\left\{\nabla_{t}^{2}+2 i k \frac{\partial}{\partial z}+\right. & \omega^{2} \mu \delta \epsilon-i\left[2 k \alpha_{1}-\omega \mu \sigma_{0}+\omega \mu b\right] \\
& \left.\times\left[\left|A_{1}\right|^{2}+\left(\begin{array}{l}
1 \\
2
\end{array}\right)\left|A_{2}\right|^{2}\right]\right\} a_{1}^{*}=0 .
\end{aligned}
$$

Phase conjugation, in the approximate sense discussed at the end of Case 3 , is possible since we can put

$$
\begin{aligned}
& \alpha_{2}=\frac{\omega \mu}{2 k}\left\{\sigma_{0}-b\left[\left(\begin{array}{l}
1 \\
2
\end{array}\right)\left|A_{1}\right|^{2}+\left|A_{2}\right|^{2}\right]\right\}, \\
& \alpha_{1}=\frac{\omega \mu}{2 k}\left\{\sigma_{0}-b\left[\left|A_{1}\right|^{2}+\left(\begin{array}{l}
1 \\
2
\end{array}\right)\left|A_{2}\right|^{2}\right]\right\} .
\end{aligned}
$$

Note that, in the nondiffusive case, $\left(\frac{1}{2}\right)=2, \alpha_{2} \neq \alpha_{1}$, i.e., each wave sees a different loss. In the case $\left|A_{2}\right|^{2} \gg\left|A_{1}\right|^{2}$ we can obtain $\alpha_{1}<0$, i.e., amplification of the weak signal. The different effective loss suffered by each wave reflects the fact that the net balance of power in each wave involves not only ohmic losses but also power that is Bragg scattered from the other wave into the wave in question.

In summary, we have explored the conditions that need be satisfied by propagation media to enable phase conjugation to occur. We find that, under certain realistic conditions involving paraxial beam propagation, phase conjugation obtains even in lossy and saturable (nonlinear) media.

This paper was delivered at the International Symposium, Huygens Principle 1690-1990, The Hague, The Netherlands, November 1990.

This research was supported by the U.S. Army Research Office, Durham, North Carolina.

\section{References}

1. A. Yariv, IEEE J. Quantum Electron. QE-14, 650 (1978).

2. R. A. Fischer, ed., Optical Phase Conjugation (Academic, New York, 1983).

3. D. M. Pepper and A. Yariv, Opt. Lett. 5, 59 (1980). 